\title{
Antenatal Magnesium Sulfate, Necrotizing Enterocolitis, and Death among Neonates $<28$ Weeks Gestation
}

\author{
Manijeh Kamyar, MD ${ }^{1} \quad$ Erin A. S. Clark, MD ${ }^{1} \quad$ Bradley A. Yoder, MD ${ }^{2}$ Michael W. Varner, MD ${ }^{1}$ \\ Tracy A. Manuck, MD ${ }^{1,3}$
}

${ }^{1}$ Division of Maternal-Fetal Medicine, Department of Obstetrics and Gynecology, University of Utah, Salt Lake City, Utah

2 Division of Neonatology, Department of Pediatrics, University of Utah, Salt Lake City, Utah

${ }^{3}$ Division of Maternal Fetal Medicine, Department of Obstetrics and Gynecology, University of North Carolina-Chapel Hill, Chapel Hill, North Carolina

Am J Perinatol Rep 2016;6:e148-e154.

\begin{abstract}
Address for correspondence Manijeh Kamyar, MD, Department of Obstetrics and Gynecology, University of Utah, 30 North Medical Drive, Suite 2B200, Salt Lake City, UT 84132 (e-mail: mkamyar@hrpregnancy.com).
\end{abstract}

\begin{abstract}
Objective This study aims to examine the relationship between antenatal magnesium sulfate $\left(\mathrm{MgSO}_{4}\right)$ and neonatal death and/or severe necrotizing enterocolitis (NEC) among infants $<28$ weeks.

Methods Secondary analysis of a multicenter randomized trial of antenatal $\mathrm{MgSO}_{4}$ versus placebo administered to women to prevent death and cerebral palsy. Neonates $<28$ weeks were included. The primary outcome was neonatal death before NICU discharge, and/or severe NEC (Bell criteria stage II/III). Neonates with and without death/ severe NEC were compared.

Results A total of 697 neonates met the criteria. Out of which 150 (21.5\%) died and/or were diagnosed with severe NEC. Antenatal $\mathrm{MgSO}_{4}$ exposure was not associated with death/severe NEC in infants $<28$ weeks. In a subgroup analysis of neonates $<26$ weeks, treatment group assignment to antenatal $\mathrm{MgSO}_{4}$ was associated with an increased odds of death/severe NEC (adjusted odds ratio: 1.90, 95\% confidence interval: 1.12-3.22, $p=0.017$ ).

Conclusions Among neonates $<26$ weeks, antenatal $\mathrm{MgSO}_{4}$ was associated with death and severe NEC. Further prospective study in larger populations is needed.
\end{abstract}

Multiple studies have investigated the use of antenatal magnesium sulfate $\left(\mathrm{MgSO}_{4}\right)$ to prevent cerebral palsy $(\mathrm{CP})$ in the offspring. ${ }^{1-4}$ The majority of these studies have shown that antenatal $\mathrm{MgSO}_{4}$ reduces the risk of gross motor dysfunction and $\mathrm{CP}$ among surviving early preterm infants. A meta-analysis and Cochrane review support these findings; thus, it is now standard of care in the United States to administer $\mathrm{MgSO}_{4}$ before an expected early preterm delivery $\left(<32\right.$ weeks). ${ }^{5,6}$ However, a few investigators have recently questioned the

received

November 11, 2015

accepted after revision

February 19, 2016
DOI http://dx.doi.org/

10.1055/s-0036-1581059. ISSN 2157-6998. standardized antenatal $\mathrm{MgSO}_{4}$ clinical administration proto$\mathrm{col}^{7}{ }^{7}$ and others are continuing to explore possible neonatal risks associated with $\mathrm{MgSO}_{4}$ use.

A recent publication reported a possible association between antenatal $\mathrm{MgSO}_{4}$ use and spontaneous intestinal perforation (SIP) among extremely low-birth-weight infants. Rattray et al performed a retrospective review of 155 extremely low-birth-weight infants $(<1,000 \mathrm{~g})$ to examine the risk of SIP and/or death in relationship to antenatal $\mathrm{MgSO}_{4}$
Copyright $\odot 2016$ by Thieme Medical Publishers, Inc., 333 Seventh Avenue, New York, NY 10001, USA. Tel: +1(212) 584-4662.
License terms

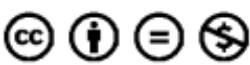


use. $^{8}$ They found a higher rate of SIP and/or death among infants who had received $\mathrm{MgSO}_{4}$ (30.4 vs. $20.5 \%$ in those without $\mathrm{MgSO}_{4}$ exposure, $p=0.28$ ); the effect was most pronounced among those delivered $<25$ weeks gestation (odds ratio [OR]: 9.7, $p<0.01$ ).

SIP is common among very premature neonates, affecting approximately $5 \%$ of extremely low-birth-weight neonates, and is associated with a 10 to $36 \%$ mortality rate. ${ }^{9,10}$ Necrotizing enterocolitis (NEC) is a separate, generally more severe gastrointestinal disorder, affecting approximately $10 \%$ of extremely low-birth-weight neonates. The mortality rate of severe NEC is 30 to $50 \%$ and significant long-term morbidity is common amongst survivors. ${ }^{11}$ Differentiation between NEC and SIP is usually based on physical examination, clinical manifestations (NEC typically more systemic), and radiographic findings (pneumatosis intestinalis is present only in NEC). These conditions can be diagnosed clinically, though intraoperative evaluation is the only way to truly distinguish between the two entities. The etiology of NEC is multifactorial, and includes bacterial proliferation and overgrowth as well as ischemic necrosis of the intestinal mucosa. ${ }^{12}$ The etiology of SIP is poorly understood but is also likely multifactorial. ${ }^{13}$ Although different processes, disruption of normal intestinal flora, motility, and/or mucosal integrity may contribute to the development of both.

We sought to examine factors associated with neonatal death and NEC, and to investigate the relationship between $\mathrm{MgSO}_{4}$ exposure, neonatal death, and NEC among infants delivered $<28$ weeks gestation.

\section{Materials and Methods}

This is a secondary analysis of a multicenter randomized controlled trial conducted by the Eunice Kennedy Shriver National Institute of Child Health and Human Development Maternal Fetal Medicine Units Network of antenatal $\mathrm{MgSO}_{4}$ versus placebo administered to women at imminent risk for preterm delivery less than 32 weeks gestation. The aim of the primary study was to investigate the role of antenatal $\mathrm{MgSO}_{4}$ in the prevention of death and $\mathrm{CP}$ in their offspring. The methods and results from the primary study have been previously published. ${ }^{4}$ Briefly, the main trial found that fetal exposure to $\mathrm{MgSO}_{4}$ did not reduce the combined risk of moderate or severe $\mathrm{CP}$ or death, but the rate of moderateto-severe CP was reduced among survivors (1.9 vs. 3.5\%; relative risk, 0.55 ; 95\% confidence interval [CI], 0.32-0.95). All participants provided written informed consent at the time of enrollment in the original study. This secondary analysis was performed on a de-identified data set, and was reviewed by our local institutional review board (IRB) and determined to be nonhuman subjects research and therefore exempt from IRB approval.

Singleton and twin infants admitted, randomized, and delivered between 23.0 and 27.9 weeks gestation were included in this secondary analysis. Enrollment occurred from December 1997 through May 2004. Infants with chromosomal abnormalities, major congenital malformations, and/or with incomplete outcomes were excluded. Gestational age was determined by the best obstetric estimate per previously published criteria. ${ }^{14}$ Infants were classified as small for gestational age if their birth weight was less than the 10th percentile based on gender- and gestational-agespecific contemporary birth norms. ${ }^{15}$ Trained research nurses obtained data on neonatal outcomes during hospitalization and at discharge. Specifically, each neonate was assessed for the presence of or history of intraventricular hemorrhage, periventricular leukomalacia, bronchopulmonary dysplasia, retinopathy of prematurity, and NEC. Additionally, charts were reviewed to determine if the neonate had one or more documented (culture proven) episode(s) of sepsis during their hospitalization. ${ }^{4}$

The primary outcome of this secondary analysis was the incidence of death before initial neonatal hospital discharge and/or diagnosis of moderate or severe (stage II or III) NEC. NEC was diagnosed based on the clinical staging system of Bell, et al. ${ }^{16}$ We also examined factors associated with neonatal death and stage II/III NEC individually. Information on SIP was not available because it was not specifically collected in the primary study.

Demographics, pregnancy characteristics, and neonatal outcomes were compared between children with and without death and/or stage II/III NEC. These univariable analyses were conducted using Student $t$-test, chi-square, and analysis of variance, as appropriate. Data were then analyzed by multivariable regression using backward regression, to test for an interaction between $\mathrm{MgSO}_{4}$ exposure and death and/or stage II/III NEC. Exposure to $\mathrm{MgSO}_{4}$ and factors with a $p$ value $<0.20$ remained in the final models. Initial covariates included in each regression model were delivery gestational age, treatment group assignment (randomization to $\mathrm{MgSO}_{4}$ or placebo), fetal sex, small for gestational age, chorioamnionitis, cesarean section, neonatal hypotension during initial resuscitation, postnatal exposure to indomethacin, neonatal sepsis, and intraventricular hemorrhage. A preplanned subgroup analysis was also performed for neonates delivered $<26$ weeks gestation. Data were analyzed using Stata version 13.1 (StataCorp LP, College Station, TX).

\section{Results}

Of 2,444 neonates randomized in the original study, 697 neonates delivered $<28$ weeks gestation met inclusion criteria for this secondary analysis (-Fig. 1). From this group, 114 babies (16.3\%) were diagnosed with NEC, including 47 with stage I, 25 with stage II, and 42 with stage III NEC. Death was increasingly likely with higher stages of NEC (8.5\% with stage I, $28.0 \%$ with stage II, and $50.0 \%$ with stage III, $p<0.001)$. A total of 111 neonates died before initial hospital discharge, at a median 13 days of life (interquartile range [IQR]: 2-37).

Overall, 150 (21.5\%) babies died and/or were diagnosed with stage II/III NEC during their initial NICU hospitalization and met criteria for the primary outcome. The 67 neonates with stage II/III NEC were initially diagnosed with NEC at a median 20 (IQR: 13-40) days of life. Neonates who died or developed stage II/III NEC were more likely to be small for gestational age, and were enrolled and subsequently delivered earlier than those 


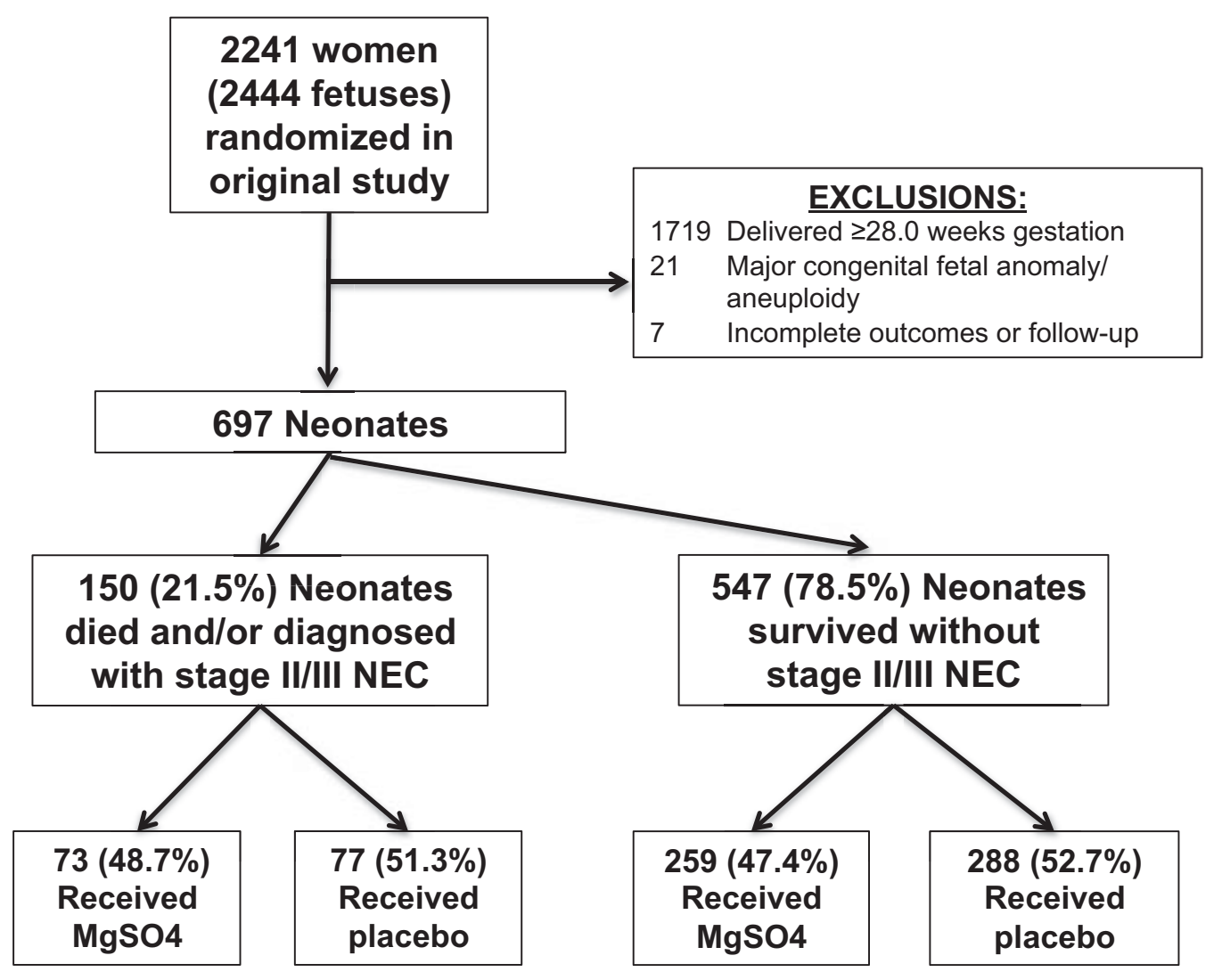

Fig. 1 Inclusion criteria.

neonatal survivors who did not have severe NEC. Demographic and baseline characteristics were otherwise similar between those with and without the adverse outcome and are compared in - Table 1. Delivery characteristics and initial neonatal outcomes are shown in - Table 2.

After adjusting for confounders, delivery gestational age, neonatal sepsis, postnatal exposure to indomethacin, male sex, and cesarean section remained associated with our primary outcome of neonatal death or stage II/III NEC ( - Table 3). In the overall cohort of neonates delivered $<28$ weeks, those randomized to antenatal $\mathrm{MgSO}_{4}$ were not at increased odds of death or stage II/III NEC. Randomization to $\mathrm{MgSO}_{4}$ was also not associated with elevated odds of the individual outcomes of death and stage II/III NEC among those delivered $<28$ weeks gestation in multivariable models (data not shown).

Overall, 293 neonates (42.0\%) were delivered less than 26.0 weeks gestation. As expected, these very premature neonates were more likely to die or develop stage II/III NEC compared with babies delivered 26.0 to 27.9 weeks (87/293, $29.7 \%$ vs. $63 / 404,15.6 \%, p<0.001)$. Of babies delivered $<26$ weeks gestation, 148 (50.5\%) were randomized to receive $\mathrm{MgSO}_{4}$ in the original study. Of these, 53/148 (35.8\%) died or developed stage II/III NEC, compared with 34/145 (23.5\%) babies who received placebo $(p=0.021)$, unadjusted OR, 1.82 (95\% CI: $1.10-3.03, p=0.021)$. In multivariable models, treatment group assignment to antenatal $\mathrm{MgSO}_{4}$ remained associated with an increased odds of death or stage III NEC (adjusted odds ratio [aOR]: $1.90,95 \% \mathrm{CI}: 1.12-3.22, p=0.017$ ), even when controlling for confounders including delivery gestational age and small for gestational age (-Table 4 ). This finding appeared to be driven primarily by an increased risk of death. In additional multivariable models, randomization to $\mathrm{MgSO}_{4}$ was associated with death with an aOR of 1.83 (95\% CI: 1.03-3.27, $p=0.040$ ). This is in contrast to the model with stage II/III NEC alone as the dependent variable, where randomization to $\mathrm{MgSO}_{4}$ was not associated with this adverse outcome (aOR: $1.38,95 \% \mathrm{CI}: 0.64-3.00, p=0.414$ ).

\section{Discussion}

We report no association between randomization to antenatal $\mathrm{MgSO}_{4}$ for fetal neuroprotection and the combined outcome of death and/or stage II/III NEC among very preterm neonates delivered $<28$ weeks gestation. In the subgroup of neonates delivered $<26$ weeks gestation, there was an association between randomization to $\mathrm{MgSO}_{4}$ and these adverse outcomes.

The reason for this association is unclear. $\mathrm{MgSO}_{4}$ readily crosses the placenta, with a high correlation between fetal and maternal $\mathrm{MgSO}_{4}$ levels. ${ }^{17}$ The time between antenatal $\mathrm{MgSO}_{4}$ exposure and NEC diagnosis at a median of 20 days of life (IQR: 13-40) suggests there is not a direct causal relationship; $\mathrm{MgSO}_{4}$ is likely one contributing "environmental" factor to the development of NEC. Various biological effects of $\mathrm{MgSO}_{4}$ may affect the integrity of the gastrointestinal tract and may contribute to the development of SIP and/or NEC. Higher $\mathrm{MgSO}_{4}$ levels are associated with intestinal dysmotility and fecal impaction among adults; it is reasonable to 
Table 1 Maternal demographic and baseline characteristics at randomization

\begin{tabular}{|l|l|l|l|}
\hline Characteristic & $\begin{array}{l}\text { NICU death or } \\
\text { stage II/III NEC } \\
N=150\end{array}$ & $\begin{array}{l}\text { Survived NICU, } \\
\text { no stage II/III NEC } \\
N=547\end{array}$ & $p$ Value \\
\hline Maternal age (y) & $26.7 \pm 6.0$ & $26.5 \pm 6.1$ & 0.756 \\
\hline Maternal prepregnancy body mass index $\left(\mathrm{kg} / \mathrm{m}^{2}\right)$ & $26.7 \pm 6.8$ & $27.5 \pm 7.3$ & 0.263 \\
\hline Married & $74(49.3)$ & $259(47.5)$ & 0.694 \\
\hline Race/ethnicity & & & 0.290 \\
\hline African-American & $72(48.0)$ & $262(47.9)$ & \\
\hline Caucasian & $54(36.0)$ & $184(33.6)$ & \\
\hline Hispanic & $24(16.0)$ & $88(16.1)$ & \\
\hline Other & $0(0)$ & $13(2.3)$ & 0.700 \\
\hline Maternal education: highest level completed & $12.0( \pm 2.3)$ & $12.0( \pm 2.3)$ & 0.797 \\
\hline No prenatal care & $9(6.0)$ & $36(6.6)$ & 0.359 \\
\hline Smoking during pregnancy & $35(23.3)$ & $148(27.1)$ & 0.832 \\
\hline Alcohol use during pregnancy & $14(9.3)$ & $48(8.8)$ & 0.153 \\
\hline Illicit drug use during pregnancy & $18(12.0)$ & $45(8.2)$ & $<0.001$ \\
\hline Gestational age at randomization (wks) & $25.2 \pm 1.0$ & $25.6 \pm 1.1$ & 0.775 \\
\hline Randomized to magnesium sulfate & $73(48.7)$ & $259(47.4)$ & 0.318 \\
\hline Preterm premature rupture of membranes & $127(84.7)$ & $480(87.8)$ & \\
\hline
\end{tabular}

Abbreviations: NICU, neonatal intensive care unit; NEC, necrotizing enterocolitis

hypothesize that this might also occur in neonates. ${ }^{18}$ Decreased intestinal motility could also result in bacterial overgrowth, leading to NEC. The immature epithelium in premature newborns is more sensitive to bacteria and bacterial translocation, which may contribute to changing the intestinal microbiome, causing intestinal damage and NEC. ${ }^{19}$ In rat models, $\mathrm{MgSO}_{4}$ has also been shown to reduce mesenteric blood flow in vivo. ${ }^{20,21}$ A reduction in mesenteric blood flow in neonates may cause vulnerability to ischemic bowel injury. As both SIP and NEC are multifactorial, the combination of an immature gastrointestinal tract, $\mathrm{MgSO}_{4}$, and other genetic or environmental risk factors may be sufficient to trigger for the development of these severe complications. We hypothesize that these adverse effects are found only among the most premature neonates due to the extreme fragility and immaturity of the gastrointestinal tract $<26$ weeks gestation. However, the exact mechanism (s) are unknown, and we are unable to investigate these etiologies using the current study design.

Our results have some similarities to those reported by Rattray and colleagues. They found a $30 \%$ incidence of SIP and/ or death during the time period when an antenatal $\mathrm{MgSO}_{4}$ for neuroprotection protocol was implemented, in contrast to SIP and/or death rates of $13 \%$ off protocol. ${ }^{8}$ Rattray included all neonates with birthweight $<1,000 \mathrm{~g}$; in contrast, our study included those delivered $<28$ weeks gestation. In both studies, however, the findings were most significant for neonates delivered $<26$ weeks gestation. We performed this subgroup analysis due to known increases in risk for NEC in infants born $<26$ weeks gestational age. ${ }^{22}$
Our findings are in contrast to other studies that have shown no association between $\mathrm{NEC}$ and antenatal $\mathrm{MgSO}_{4}$. Ghidini et al performed a retrospective cohort study comparing the rate of $\mathrm{MgSO}_{4}$ exposure before birth among 23 infants diagnosed with NEC to 46 controls, and found a similar proportion of babies were exposed to $\mathrm{MgSO}_{4}$ in each group (30 vs. 39\%, $p=0.4$ ). ${ }^{23}$ In another retrospective cohort study of 401 infants delivered 23 to 34 weeks gestation, approximately half were exposed to $\mathrm{MgSO}_{4}$, and there was no apparent relationship between $\mathrm{MgSO}_{4}$ exposure and $\mathrm{NEC}^{24}$ Although neither study demonstrated an association between $\mathrm{MgSO}_{4}$ and NEC, these studies were retrospective and nonrandomized, included infants delivered across a large gestational age range (with a lower overall incidence of NEC), and were ultimately underpowered.

Although prenatal indomethacin exposure classically has been associated with an increased risk of NEC, we were unable to confirm those findings in the present study. Historically, the association between postnatal indomethacin exposure and NEC has been less consistent, and some reports have endorsed a protective association while others have noted a potentially negative association. ${ }^{26,27}$ In our study, we found a reduced likelihood of stage II/III NEC among those neonates delivered $<28$ weeks who were exposed to postnatal indomethacin (aOR: 0.63, 95\% CI: 0.42-0.94).

This study has several strengths. This was a large, prospectively collected cohort. All mothers randomized to receive $\mathrm{MgSO}_{4}$ did so per study protocol (with set loading and maintenance dosing, and uniform conditions for drug initiation and discontinuation). All neonates were evaluated in a standardized fashion by trained research nurses and 
e152 Antenatal $\mathrm{MgSO}_{4}$, NEC, and Death among Neonates < 28 Wks Gestation Kamyar et al.

Table 2 Delivery characteristics and initial neonatal outcomes. Unless otherwise specified, data are listed as $n(\%)$

\begin{tabular}{|c|c|c|c|}
\hline Characteristic & $\begin{array}{l}\text { NICU death and/or } \\
\text { stage II/III NEC } \\
N=150\end{array}$ & $\begin{array}{l}\text { Survived NICU, } \\
\text { no stage II/III NEC } \\
N=547\end{array}$ & $p$ Value \\
\hline Gestational age at delivery (wks) & $25.8 \pm 1.1$ & $26.3 \pm 1.0$ & $<0.001$ \\
\hline Antenatal exposure to indomethacin & $11(7.3)$ & $47(8.6)$ & 0.621 \\
\hline Received antenatal corticosteroids & $145(96.7)$ & $528(96.5)$ & 0.934 \\
\hline Chorioamnionitis & $19(12.7)$ & $97(17.7)$ & 0.140 \\
\hline Magnesium sulfate infusing at delivery ${ }^{\mathrm{a}}$ & $41 / 73(56.2)$ & $152 / 259(58.7)$ & 0.700 \\
\hline Mean total amount $(\mathrm{g})$ of magnesium sulfate received $( \pm \mathrm{SD})^{\mathrm{a}}$ & $33.0 \pm 19.8$ & $33.9 \pm 18.2$ & 0.595 \\
\hline Mean cord blood magnesium sulfate level, mEq/L ( \pm SD) ${ }^{\mathrm{b}}$ & $2.64 \pm 1.1$ & $2.67 \pm 0.8$ & 0.890 \\
\hline Cesarean section & $77(51.3)$ & $256(46.8)$ & 0.325 \\
\hline Male fetus & $87(58.0)$ & $269(49.2)$ & 0.056 \\
\hline Birthweight $(\mathrm{g})$ & $757 \pm 164$ & $872 \pm 181$ & $<0.001$ \\
\hline Small for gestational age & $20(13.3)$ & $24(4.4)$ & $<0.001$ \\
\hline Median 1-min Apgar score (IQR) & $4(2-6)$ & $5(3-7)$ & $<0.001$ \\
\hline Median 5-min Apgar score (IQR) & $7(5-8)$ & $7(6-8)$ & $<0.001$ \\
\hline Neonatal hypotension in the delivery room & $9(6.0)$ & $21(3.8)$ & 0.248 \\
\hline Neonatal hypotension requiring treatment at any time & $117(78.0)$ & $183(33.5)$ & $<0.001$ \\
\hline Patent ductus arteriosus requiring therapy & $50(33.3)$ & $179(32.7)$ & 0.888 \\
\hline Postnatal exposure to indomethacin & $57(38.0)$ & $234(42.8)$ & 0.293 \\
\hline Culture-proven sepsis & $81(54.0)$ & $183(33.5)$ & $<0.001$ \\
\hline Neonatal seizures (suspected or confirmed) & $14(9.3)$ & $22(4.0)$ & 0.009 \\
\hline Any intraventricular hemorrhage & $41(33.3)$ & $159(29.5)$ & 0.403 \\
\hline Severe intraventricular hemorrhage (grade III or IV) & $15(12.2)$ & $31(5.8)$ & 0.011 \\
\hline
\end{tabular}

Abbreviations: IQR, interquartile range; NEC, necrotizing enterocolitis; NICU, neonatal intensive care unit; SD, standard deviation.

${ }^{a} \mathrm{O} 332$ neonates randomized to receive $\mathrm{MgSO}_{4}$.

b $\mathrm{Of} 170$ neonates randomized to receive $\mathrm{MgSO}_{4}$ with cord blood $\mathrm{MgSO}_{4}$ levels available.

physicians. We had comprehensive pregnancy information, and were able to incorporate these data into our analyses and multivariable models.

This study should be interpreted with several limitations in mind. We were limited by data collected at the time of the

Table 3 Multivariable regression results for association with neonatal death and/or stage II/III NEC for all 697 neonates delivered $<28$ wks

\begin{tabular}{|l|l|l|}
\hline & OR (95\% Cl) & $p$ Value \\
\hline $\begin{array}{l}\text { Delivery gestational age } \\
\text { (per 1 wk increment) }\end{array}$ & $0.67(0.56-0.81)$ & $<0.001$ \\
\hline Neonatal sepsis & $2.14(1.45-3.16)$ & $<0.001$ \\
\hline Small for gestational age & $2.88(1.49-5.57)$ & 0.002 \\
\hline $\begin{array}{l}\text { Postnatal exposure to } \\
\text { indomethacin }\end{array}$ & $0.63(0.42-0.94)$ & 0.023 \\
\hline Male fetus & $1.34(0.91-1.96)$ & 0.134 \\
\hline $\begin{array}{l}\text { Randomized to receive } \\
\text { magnesium sulfate }\end{array}$ & $1.01(0.69-1.47)$ & 0.965 \\
\hline
\end{tabular}

Abbreviations: $\mathrm{Cl}$, confidence interval; NEC, necrotizing enterocolitis; OR, odds ratio. initial study, and therefore were unable to examine SIP as an outcome. As with all unplanned secondary analyses, there is a possibility that reported findings may represent spurious results. Although NEC and SIP are different processes, intraoperative evaluation is the only way to truly distinguish between the two entities, and it is possible that there was some degree of diagnostic overlap in this dataset. Additionally, we had limited information regarding specifics of the

Table 4 Multivariable regression results. Shown are factors associated with neonatal death and/or stage II/III NEC among 293 neonates delivered $<26$ wks

\begin{tabular}{|l|l|l|}
\hline & OR (95\% CI) & $p$ Value \\
\hline $\begin{array}{l}\text { Delivery gestational age } \\
\text { (per 1 wk increment) }\end{array}$ & $0.52(0.31-0.88)$ & 0.015 \\
\hline $\begin{array}{l}\text { Randomized to receive } \\
\text { magnesium sulfate }\end{array}$ & $1.90(1.12-3.22)$ & 0.017 \\
\hline Small for gestational age & $2.47(1.04-5.85)$ & 0.041 \\
\hline Male fetus & $1.47(0.87-2.471)$ & 0.153 \\
\hline
\end{tabular}

Abbreviations: $\mathrm{Cl}$, confidence interval; NEC, necrotizing enterocolitis; OR, odds ratio. 
care received in the NICU after delivery. Although we were able to account for some postnatal risk factors previously associated with NEC (e.g., indomethacin exposure, hypotension), we were unable to control for others (e.g., neonatal steroid exposure, antibiotic exposure, breast milk exposure, and probiotic use). Lack of important neonatal covariables may significantly influence the results. Due to the enrollment criteria for the primary study, the vast majority of children were delivered following PPROM. It is unclear whether these results are applicable to a more general preterm population. However, prior studies examining outcomes stratified by preterm birth indication suggest similar outcomes regardless of delivery indication. ${ }^{28,29}$

Perhaps our most significant limitation is that these data are not contemporary. The original trial enrolled women and infants between December 1997 and May 2004. Since that time, there have been decreases in rates of NEC, as well as improvements in management of NEC. However, the incidence of NEC in our study is similar to most contemporary data. Our study population was remarkable for an overall NEC incidence of $16.4 \%$, and a stage II/III NEC incidence of $9.6 \%$. Recent reports from other centers describe the incidence of NEC to be 3 to $15 \%,{ }^{30,31}$ and unpublished data from the University of Utah (provided by author B. A. Y.) from 2011 to 2014 show an overall NEC rate of $10 \%$ among infants born $<28$ weeks. It should be noted that a raw comparison of NEC incidence does not account for the competing outcome of death.

The study by Rattray et al, taken in combination with our findings, raise concern regarding the use of $\mathrm{MgSO}_{4}$ at the earliest gestational ages, at least in the standardized dosage utilized in our parent study. However, the study design and level of evidence provided here is insufficient to, and should not, change current clinical practice. Infants born $<26$ weeks are those at highest risk for $\mathrm{CP}$, and thus have the highest potential neuroprotective benefit from antenatal $\mathrm{MgSO}_{4}$ use. The authors strongly feel that in lieu of more rigorous evidence, obstetrical care providers should continue to administer antenatal $\mathrm{MgSO}_{4}$ to all eligible patients per local protocols. These findings urgently need to be verified in more contemporary cohorts (ideally, prospectively collected). In future investigations, careful attention should be paid to maternal-fetal and neonatal pharmacokinetics, and associated outcomes to determine if there is a dose-response effect. In the meantime, obstetrical care providers should ensure that neonatologists are aware of antenatal $\mathrm{MgSO}_{4}$ use, and should have a high index of suspicion for severe gastrointestinal complications when caring for neonates delivered $<26$ weeks gestation, particularly if exposed to antenatal $\mathrm{MgSO}_{4}$.

\section{Acknowledgments}

The contents of this report represent the views of the authors and do not represent the views of the NICHD MFMU Network. This study was funded by the Eunice Kennedy Shriver National Institute of Child Health and Human Development 5K23HD067224 (Dr. Manuck) and K23HD061910 (Dr. Clark).

\section{References}

1 Marret S, Marpeau L, Zupan-Simunek V, et al; PREMAG trial group. Magnesium sulphate given before very-preterm birth to protect infant brain: the andomized controlled PREMAG trial. BJOG 2007; 114(3):310-318

2 Magpie Trial Follow-Up Study Collaborative Group. The Magpie Trial: a andomized trial comparing magnesium sulphate with placebo for pre-eclampsia. Outcome for children at 18 months. BJOG 2007;114(3):289-299

3 Crowther CA, Hiller JE, Doyle LW, Haslam RR; Australasian Collaborative Trial of Magnesium Sulphate (ACTOMg SO4) Collaborative Group. Effect of magnesium sulfate given for neuroprotection before preterm birth: a randomized controlled trial. JAMA 2003; 290(20):2669-2676

4 Rouse DJ, Hirtz DG, Thom E, et al; Eunice Kennedy Shriver NICHD Maternal-Fetal Medicine Units Network. A randomized, controlled trial of magnesium sulfate for the prevention of cerebral palsy. N Engl J Med 2008;359(9):895-905

5 Costantine MM, Weiner SJ; Eunice Kennedy Shriver National Institute of Child Health and Human Development MaternalFetal Medicine Units Network. Effects of antenatal exposure to magnesium sulfate on neuroprotection and mortality in preterm infants: a meta-analysis. Obstet Gynecol 2009;114(2 Pt 1): 354-364

6 Nguyen TMN, Crowther CA, Wilkinson D, Bain E. Magnesium sulphate for women at term for neuroprotection of the fetus. Cochrane Database Syst Rev 2013;2:CD009395

7 Pryde PG, Mittendorf R. Using prophylactic, but not tocolytic, magnesium sulfate to reduce cerebral palsy related to prematurity: what dose, and what about infant mortality? J Perinat Med 2011;39(4):375-378

8 Rattray BN, Kraus DM, Drinker LR, Goldberg RN, Tanaka DT, Cotten $\mathrm{CM}$. Antenatal magnesium sulfate and spontaneous intestinal perforation in infants less than 25 weeks gestation. J Perinatol 2014;34(11):819-822

9 Wadhawan R, Oh W, Hintz SR, et al; NICHD Neonatal Research Network. Neurodevelopmental outcomes of extremely low birth weight infants with spontaneous intestinal perforation or surgical necrotizing enterocolitis. J Perinatol 2014;34(1):64-70

10 Blakely ML, Tyson JE, Lally KP, et al; NICHD Neonatal Research Network. Laparotomy versus peritoneal drainage for necrotizing enterocolitis or isolated intestinal perforation in extremely low birth weight infants: outcomes through 18 months adjusted age. Pediatrics 2006;117(4):e680-e687

11 Neu J, Walker WA. Necrotizing enterocolitis. N Engl J Med 2011; 364(3):255-264

12 Neu J. Necrotizing enterocolitis: the mystery goes on. Neonatology 2014;106(4):289-295

13 Gordon PV, Herman AC, Marcinkiewicz M, Gaston BM, Laubach VE, Aschner JL. A neonatal mouse model of intestinal perforation: investigating the harmful synergism between glucocorticoids and indomethacin. J Pediatr Gastroenterol Nutr 2007;45(5):509-519

14 Carey JC, Klebanoff MA, Hauth JC, et al; National Institute of Child Health and Human Development Network of Maternal-Fetal Medicine Units. Metronidazole to prevent preterm delivery in pregnant women with asymptomatic bacterial vaginosis. $\mathrm{N}$ Engl J Med 2000;342(8):534-540

15 Olsen IE, Groveman SA, Lawson ML, Clark RH, Zemel BS. New intrauterine growth curves based on United States data. Pediatrics 2010;125(2):e214-e224

16 Bell MJ, Ternberg JL, Feigin RD, et al. Neonatal necrotizing enterocolitis. Therapeutic decisions based upon clinical staging. Ann Surg 1978;187(1):1-7

17 Hallak M, Berry SM, Madincea F, Romero R, Evans MI, Cotton DB. Fetal serum and amniotic fluid magnesium concentrations with maternal treatment. Obstet Gynecol 1993;81(2):185-188 
e154 Antenatal $\mathrm{MgSO}_{4}, \mathrm{NEC}$, and Death among Neonates $<28$ Wks Gestation Kamyar et al.

18 Lu JF, Nightingale CH. Magnesium sulfate in eclampsia and preeclampsia: pharmacokinetic principles. Clin Pharmacokinet 2000; 38(4):305-314

19 Tanner SM, Berryhill TF, Ellenburg JL, et al. Pathogenesis of necrotizing enterocolitis: modeling the innate immune response. Am J Pathol 2015;185(1):4-16

20 Kemp PA, Gardiner SM, March JE, Rubin PC, Bennett T. Assessment of the effects of endothelin- 1 and magnesium sulphate on regional blood flows in conscious rats, by the coloured microsphere reference technique. Br J Pharmacol 1999;126(3):621-626

21 Kemp PA, Gardiner SM, March JE, Bennett T, Rubin PC. Effects of NGnitro-L-arginine methyl ester on regional haemodynamic responses to MgSO4 in conscious rats. Br J Pharmacol 1994;111(1):325-331

22 Rees CM, Eaton S, Pierro A. National prospective surveillance study of necrotizing enterocolitis in neonatal intensive care units. J Pediatr Surg 2010;45(7):1391-1397

23 Ghidini A, Espada RA, Spong CY. Does exposure to magnesium sulfate in utero decrease the risk of necrotizing enterocolitis in premature infants? Acta Obstet Gynecol Scand 2001;80(2):126-129

24 Hammers AL, Sanchez-Ramos L, Kaunitz AM. Antenatal exposure to indomethacin increases the risk of severe intraventricular hemorrhage, necrotizing enterocolitis, and periventricular leukomalacia: a systematic review with metaanalysis. Am J Obstet Gynecol 2015;212(4):505.e1-505.e13

25 Sharma R, Hudak ML, Tepas JJ III, et al. Prenatal or postnatal indomethacin exposure and neonatal gut injury associated with isolated intestinal perforation and necrotizing enterocolitis. J Perinatol 2010;30(12):786-793

26 Yee WH, Soraisham AS, Shah VS, Aziz K, Yoon W, Lee SK; Canadian Neonatal Network. Incidence and timing of presentation of necrotizing enterocolitis in preterm infants. Pediatrics 2012; 129(2):e298-e304

27 Elimian A, Verma R, Ogburn P, Wiencek V, Spitzer A, Quirk JG. Magnesium sulfate and neonatal outcomes of preterm neonates. J Matern Fetal Neonatal Med 2002;12(2):118-122

28 Kimberlin DF, Hauth JC, Owen J, et al. Indicated versus spontaneous preterm delivery: An evaluation of neonatal morbidity among infants weighing $<=1000$ grams at birth. Am J Obstet Gynecol 1999;180(3 Pt 1):683-689

29 Bottoms SF, Paul RH, Mercer BM, et al. Obstetric determinants of neonatal survival: antenatal predictors of neonatal survival and morbidity in extremely low birth weight infants. Am J Obstet Gynecol 1999;180(3 Pt 1):665-669

30 Stoll BJ, Hansen NI, Bell EF, et al; Eunice Kennedy Shriver National Institute of Child Health and Human Development Neonatal Research Network. Neonatal outcomes of extremely preterm infants from the NICHD Neonatal Research Network. Pediatrics 2010;126(3):443-456

31 Su BH, Hsieh WS, Hsu CH, Chang JH, Lien R, Lin CH; Premature Baby Foundation of Taiwan (PBFT). Neonatal outcomes of extremely low birth weight infants from Taiwan: comparison with Canada, Japan and the USA. Pediatr Neonatol 2015;56(1):46-52 